Ann. Génét. Sél. anim., I977, 9 (2), 247-250.

\title{
Relations entre la croissance et l'activité en open-field chez la Poule \\ II. - Effet de la sélection pour le poids vif sur l'activité en open-field
}

\author{
J. M. FAURE et F. H. RICARD \\ avec la collaboration technique de F. Morozeau \\ Station expérimentale d'Aviculture du Magneraud, I.N.R.A., \\ B.P. 52, I770o Surgères (France)
}

\begin{abstract}
Résumé
L'étude de l'activité en open-field dans 4 souches de la même origine génétique, sélectionnées pour leur courbe de croissance, montre que la sélection pour un poids juvénile élevé entraîne une augmentation de l'activité en open-field. Par contre, la sélection sur le poids adulte n'a une influence que sur l'exploration. Ces résultats confirment l'hypothèse selon laquelle les résultats inverses observés dans deux souches sélectionnées en open-field sont dus à une dérive génétique.
\end{abstract}

\section{Introduction}

Nous avons vu dans la première partie de cet article (FAURE, I977) que la sélection pour l'activité en open-field s'était accompagnée d'une augmentation du poids dans la lignée inactive (émotive) (FAURE et FOLMER, I975, FAURE, I975), comparée à la lignée active. Ces résultats sont en contradiction avec ceux de BrowN et Nestor (1973, I974 $a$ et b), montrant que les animaux les plus sensibles aux stress ont le poids le plus faible. Pour expliquer cette contradiction, nous avons supposé que la différence de poids entre les deux souches était due à une dérive génétique due aux faibles effectifs utilisés pour la reproduction à chaque génération. Afin de vérifier cette hypothèse, nous avons mesuré l'activité en open-field de 4 souches sélectionnées pour la forme de leur courbe de croissance (RICARD, I975). 


\section{Matériel et méthodes}

\section{I. - Animaux}

Ces souches ont été décrites en détail précédemment (RICARD, I975). A partir d'une population témoin, dite Bresse-Pile, quatre souches ont été sélectionnées pour les combinaisons suivantes:

Souche X-II : poids à 8 semaines FAIBLE et poids adulte FORT Souche X-22 : poids à 8 semaines FORT et poids adulte FAIBLE Souche X-33 : poids à 8 semaines FORT et poids adulte FORT Souche X-44: poids à 8 semaines FAIBLE et poids adulte FAIBLE

La présente expérience a porté sur r 65 poussins de chacune de ces souches, poussins nés en février-mars I975.

\section{2. - Méthode expérimentale}

La mesure a été faite dans un open-field circulaire, divisé en 6 secteurs par 6 rayons lumineux aboutissant à des cellules photo-électriques. Les paramètres mesurés sont la latence (temps que met l'animal pour couper le premier rayon lumineux), l'activité (nombre de rayons coupés en roo secondes) et l'exploration (nombre de secteurs visités en roo secondes).

La latence peut au maximum être égale à roo secondes. Si elle atteint cette valeur, le test est arrêté et les autres paramètres (activité et exploration) sont considérés comme étant égaux à o. Si l'animal coupe un faisceau lumineux avant la fin de ce temps, il dispose à nouveau de roo secondes pour se déplacer (FAURE, FOLMER, I975).

\section{Résultats et discussion}

Les moyennes par souche pour les trois paramètres du comportement en open-field (latence, activité, exploration) sont indiquées dans le tableau I.

L'effet souche, pris globalement, est significatif, sauf pour la latence. Dans le tableau 2, nous indiquons les résultats d'une analyse de variance où sont pris en considération deux facteurs de variation : le poids juvénile (fort ou faible) et le poids adulte (fort ou faible).

L'effet le plus marqué est celui de la sélection pour le poids juvénile. Le poids adulte n'a une influence que sur l'exploration. L'interaction entre poids juvénile et poids adulte est toujours significative.

Les animaux les plus lourds à 8 semaines (X-22 et $\mathrm{X}-33)$ ont une latence plus courte, une activité et une exploration plus élevées. Ce sont donc les moins émotifs. Ce résultat est en accord avec les données de Brown et NESTOR mais va en sens contraire de ceux observés au Magneraud dans nos lignées actives et inactives sélectionnées à partir d'une souche de type Cornish (FAURE, I977). Le contexte génétique différent et le niveau de performance (la population "Bresse-Pile " est très 


\section{TABLEAU I}

Paramètres du comportement en open-field de souches de poulets ayant des courbes de croissances différentes (moyenne par souche) Parameters for behaviour in open field of chicken lines with different growth curves (line average)

\begin{tabular}{|c|c|c|c|c|c|c|}
\hline \multirow{2}{*}{$\begin{array}{c}\text { Paramètre } \\
\text { étudié }\end{array}$} & \multicolumn{5}{|c|}{ Souche } & \multirow{2}{*}{$\begin{array}{l}\text { Test global } \\
\text { effet souche }\end{array}$} \\
\hline & & & & & & \\
\hline Latence . . . & $4^{8,4^{8}}$ & 43,72 & $4^{0,35}$ & $4^{8,29}$ & 52,07 & NS \\
\hline Activité $\cdot \cdot \cdot \cdot$ & 4,73 & 7,07 & 6,08 & 4,85 & 6,13 & 0,05 \\
\hline Exploration . . & $\mathrm{I}, 65$ & $2,4^{\circ}$ & 2,01 & $\mathrm{I}, 82$ & 2,22 & O,OI \\
\hline
\end{tabular}

TABLEAU 2

Analyse de variance mettant en évidence les effets de la sélection pour le poids à 8 semaines et pour le poids adulte sur le comportement en open-field

Variance analysis showing selection effect for 8 weeks weight and adult weight on open-field behaviour

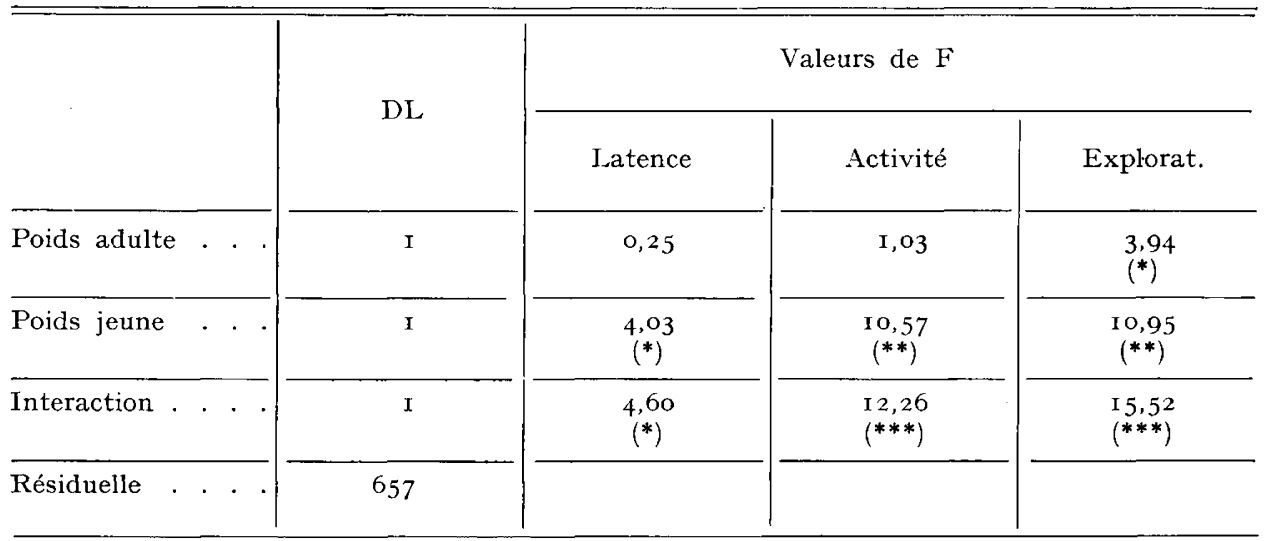

(*) Valeur de $\mathrm{F}$ significative au seuil de 0,05 ;

$(* *)$ valeur de $\mathrm{F}$ significative au seuil o,or;

$(* * *)$ valeur de $\mathrm{F}$ significative au seuil o,oor.

légère par rapport au Cornish) peuvent expliquer ces variations. Mais ces résultats confirment aussi l'hypothèse (FAURE, I977) selon laquelle la relation animaux plus lourds - animaux plus émotifs (moins actifs) est due à une dérive génétique et non à une corrélation positive entre croissance et émotivité. 


\section{Summary}

Relationship between growth and open-field activity in the hen. II. - Effect of selection for live weight on open field activity

The study of activity in open-field in 4 strains of the same origin, selected for their growth curve, shows that selection for a high weight of young birds leads to an increase of activity in open-field. On the other hand, selection on adult weight has an effect only on exploration. These results confirm the hypothesis stating that the opposite results observed in two strains selected in open-field are due to a genetical drift.

\section{Références bibliographiques}

Brown K. I., Nestor K. E., i973. Some physiological responses of turkeys selected for high and low adrenal response to cold stress. Poultry Science, 52, 1948-1954.

Brown K. I., Nestor K. E., 1974a. Implications of selection for high and low adrenal response to stress. Poultry Science, 52, I 297 -1 306.

Brown K. I., Nestor K. E., I $974 b$. Effect of stress on rate of gain and feed conversion of Ohio low and high adrenal response turkey lines. Ohio Agric. Res. Develop.Center, Res. Sum mary, 80, 4-6.

FAURE J. M., 1975. Étude des liaisons entre comportement en open-field et émotivité chez le jẹune poussin. Ann. Génét. Sél. anim., 7, 197-204.

FAURE J. M., 1977. Relations entre la croissance et l'activité en open-field: I - Effet de la sélection pour l'activité en open-field sur la croissance en poids et la conformation. Ann. Génét. Sél. anim., 9.

Faure J. M., Folmer J. C., 1975. Étude génétique de l'activité précoce en open-field du jeune poussin. Ann. Génét. Sél. anim., 7, 197-204.

Ricard F. H., I975. Essai de sélection sur la forme de la courbe de croissance chez le Poulet. Ann. Génét. Sél. anim., 7, 427-443. 